\title{
A Disposable Power Source in Resource-limited Environments: A Paper- based Biobattery Generating Electricity from Wastewater
}

\author{
Arwa Fraiwan ${ }^{\mathrm{a}}$, Landen Kwan ${ }^{\mathrm{b}}$ and Seokheun Choi ${ }^{*}$
}

${ }^{a}$ Bioelectronics \& Microsystems Laboratory, Department of Electrical \& Computer Engineering, State University of New York-Binghamton, Binghamton, NY 13902, USA

*Corresponding Author. Email: sechoi@ binghamton.edu, Fax: 1-607-777-4464

${ }^{\mathrm{b}}$ Queensborough Community College, New York, USA

Abstract: We report a novel paper-based biobattery which generates power from microorganismcontaining liquid derived from renewable and sustainable wastewater which is readily accessible in the local environment. The device fuses the art of origami and the technology of microbial fuel cells (MFCs) and has the potential to shift the paradigm for flexible and stackable paper-based batteries by enabling exceptional electrical characteristics and functionalities. 3D, modular, and retractable battery stack is created from (i) 2D paper sheets through high degrees of folding and (ii) multifunctional layers sandwiched for MFC device configuration. The stack is based on ninja star-shaped origami design formed by eight MFC modular blades, which is retractable from sharp shuriken (closed) to round frisbee (opened). The microorganism-containing wastewater is added into an inlet of the closed battery stack and it is transported into each MFC module through patterned fluidic pathways in the paper layers. During operation, the battery stack is transformed into the round frisbee to connect eight MFC modules in series for improving the power output and simultaneously expose all air-cathodes to the air for their cathodic reactions. The device generates desired values of electrical current and potential for powering an LED for more than $20 \mathrm{~min}$. 
Keywords: Disposable power source, microbial fuel cells, paper-based batteries, resource limited environments, wastewater

\section{Introduction}

Disposable lab-on-a-chip (LOC) devices have recently emerged as a new paradigm for clinical diagnostics and monitoring disease states. ${ }^{1-3}$ The devices incorporate advanced micro-sized biosensors and microfluidics, which require only small reagent volume, reduced size, and minimized power consumption. ${ }^{3}$ They also offer many other advantages including short reaction time, versatile designs, multifunctional system integration, and highly paralleled operation with small footprints, thereby enabling portability and mobility for effective and rapid point-of-care (POC) testing even in challenging field conditions where laboratory infrastructure, equipment and trained personnel, as well as access to clean water are not available. ${ }^{1-3}$ A recent advance in the LOC devices has greatly enhanced POC diagnostic performance and the technologies have been successfully introduced into the market. ${ }^{4,5}$ However, there has been a significant challenge in realizing a truly stand-alone and self-sustainable diagnostic platform that does not rely on a competent laboratory service. The key challenge is to develop a miniaturized power source for powering those POC devices. ${ }^{6}$ Power autonomy is one of the most critical requirements for the POC diagnostics that can work independently and self-sustainably in limited-resource and remote regions, where the stable electrical supply is not accessible. Even standard batteries can be problematic in those areas in terms of economic and environmental impacts. Also, conventional energy harvesting technologies (e.g. solar, thermal, mechanical, chemical energy) are too overqualified and expensive as a power source for single-use, disposable POC tests, requiring relatively small power consumption only for a couple of minutes. Therefore, there is a need for 
continuous effort towards the development of such power sources with disposability, low-cost, minimum environmental issues and accessibility in resource-limited settings.

Biomass can be one of the excellent energy harvesting sources readily available even in resource limited environments. ${ }^{7}$ Typically, microbial fuel cells (MFCs) have been used as energy transducers that convert the biological energy in biomass directly into electricity via microbial metabolism. ${ }^{8-10}$ Microorganisms oxidize organic fuels, completing respiration by transferring electrons to the device electrode. ${ }^{11,12}$ The organic fuel for microorganisms can be any type of biodegradable substrate including wastewater, urine, or soiled water in a puddle. In addition, river, ocean or pond water generally host various microorganisms that can transfer electrons via metabolism to an external electrode. For this reason, MFCs have been regarded as a promising energy technique in developing countries and the concept of the MFC has been validated by successful demonstration of large-scale system as prototypes of large power sources or energyefficient wastewater treatment technology. ${ }^{13-15}$

Miniaturizing MFC devices is also an interesting approach for potentially powering POC diagnostic tools due to easy accessibility in those regions, low-cost, and environmentally friendly features. ${ }^{16-20}$ However, the promise of this technology has not yet been translated as practical POC applications, because (i) even small-scale MFCs require a relatively long start-up time to accumulate and acclimate microorganisms on the anodic surface (several hours to days), (ii) their device configuration is complicated with necessary multifunctional parts (anode, cathode, and ion exchange membrane) along with microfluidic tubings for liquid inlets/outlets, and (iii) their operation requires additional power/equipment to continuously inject organic fuels. In order to overcome those limitations, Choi group has recently pioneered the novel micro-sized MFC platform on paper substrate as a potential power source for disposable POC devices. ${ }^{21-27}$ The paper-based MFC allowed for rapid adsorption of microorganism-containing solution through capillary force of paper and immediate microbial cells' attachment to the electrode, leading to a 
very short start-up time. Therefore, the device rapidly generated power with a small amount of microorganism-containing liquid. Moreover, the MFC configuration became simplified by using paper substrates because microfluidic channels/chambers can be easily patterned with hydrophobic wax and the 3D device structure can be constructed by applying origami techniques. In addition, no external pump/tubing were necessary to operate the device because paper has the ability to wick fluids through capillary action. If (i) a desired power output for actual applications is obtained through a fundamental device level breakthrough of this conceptual prototype and (ii) wastewater in the local environment can be a real energy source for its operation, then this novel device can be replicated, laying a foundation to explore the viability of this power source as a practical and accessible power supply even in difficult-to-reach, dangerous and/or extremely remote locations.

In this work, we created an origami battery in which eight paper-based MFC modules were stacked in series (Fig. 1). The device was based on ninja star-shaped design formed by eight modular blades, which is retractable from sharp shuriken (closed) (Fig 1a) to round frisbee (opened) (Fig. 1b). Each blade had 3D MFC structure (i.e. anode, proton exchange membrane, and air-cathode) created from 2D sheets through high degrees of folding along predefined creases. The microorganism-containing solution was added into an inlet of the closed battery stack (sharp shuriken), through which it was transported into each MFC module. During operation, the battery stack was transformed into the round frisbee to connect eight MFC modules in series for improving power output and simultaneously expose all air-cathodes to the air for their cathodic reactions. The battery stack generated desired values of electrical current and potential for powering a red LED even with readily prepared wastewater sample available for on-site operation in low resource settings and economically challenged regions of the world. 


\section{Experimental}

We created a three-dimensionally folded paper MFC stack which was capable of transforming its shape from the sharp shuriken to the round frisbee or vise-versa. The stack was composed of the eight MFC modules. With the shuriken shape, individual MFCs were disconnected and their inlets were positioned in the center for easy sample introduction. When the stack was opened for the round frisbee shape, all MFCs were connected in series and their cathodic parts were maximally exposed to the air. This miniaturized paper-based power source could lead to a very practical application by using wastewater in the local environment as the electron donor and allowing the use of freely-available oxygen in the air as the electron acceptor.

\subsection{Paper-based MFC modules}

Each MFC module was fabricated by sandwiching five functional layers: (a) air-cathodic layer on paper (Whatman $\# 1$, pore size: $10 \mu \mathrm{m}$ ), (b) paper proton exchange membrane (PEM) (Whatman $\# 410$, pore size: $1 \mu \mathrm{m}$ ), (c) paper reservoir (Whatman \#1, pore size: $10 \mu \mathrm{m}),(\mathrm{d})$ carbon cloth anode (CCP 10, Fuel Cell Earth LLC.), and (e) anodic paper layer. Fig. 2 shows the fabrication and folding processes for individual MFC modules and the detailed schematic diagram of the structure with their operating principle. (Origami-Instructions.com provides the detailed origami instruction for the ninja star). First, the hydrophilic zones with hydrophobic wax boundaries were made on Whatman\#1 filter paper for (a) cathodic and (e) anodic layers by using a commercially-available solid-wax printer (Xerox phaser printer). Then, the wax-printed paper was heated at $150^{\circ} \mathrm{C}$ for 2 min to let the wax melt and penetrate through the paper to form the hydrophobic boundaries, which defined a hydrophilic region and avoided liquid leakage to outside of the device during wet-out. The paper was then cut by using the laser machine (Universal Laser System, VLS 3.5) into the shape shown in Fig 2a (i). Fig. 2a (ii) shows that the left lower half along the predefined crease \#1 was folded back to the right lower half which then was folded up along the predefined 
crease \#2. The patterned rhombic carbon cloth anode (height: $1.5 \mathrm{~cm}$, bottom side length:3.5cm) was exposed to oxygen plasma for $1 \mathrm{~min}$ for hydrophilization and attached on top of the right part of the paper substrate by using an adhesive spray (3M Super 77 Spray Adhesive) (Fig. 2a (iii)). Additional wax-patterned reservoir layer (Whatman \#1) was put over the anode (Fig. 2a (iv)). This reservoir was to hold the microorganism-containing liquid for an extended time during the MFC operation. Then, an activated carbon based air-cathode was constructed on the left part of the paper substrate. After nickel was first sprayed as a structural support and current collector (Fig. 2a (v)), the activated carbon catalysts (CABOT Corporation) $\left(15 \mathrm{mg} / \mathrm{cm}^{2}\right)$ with binder solution (5 wt\% Nafion solution, DI water, and isopropanol) was applied (Fig. 2a (vi)). The detailed procedure for the air-cathode on paper was presented in our previous report. ${ }^{21}$ Due to the porous structure of the paper substrate, the other side of the paper allowed the oxygen to reach the aircathode to complete the redox reactions for the MFC operation. Copper tape $\left(3 \mathrm{M}^{\mathrm{TM}}\right.$ copper conductive tape) was used to provide an electrical contact to the anode. Whatman \#410 with very small pore size $(1 \mu \mathrm{m})$ was additionally attached on top of the reservoir layer for the use an efficient and inexpensive PEM (Fig. 2a (vii)). The smaller pore size than that of the bacterial cells prevented the cells from being transported to the cathode while promoting movement of the protons generated from microbial metabolism in the anodic compartment. ${ }^{28,29}$ The paper PEM also improved the proton traveling efficiency by sucking the ionic liquid of the microorganismcontaining solution. While there are only two electrical contact parts in the MFC module for the anode and cathode, all edges of the MFC blade were covered with the copper tapes for better electrical connection upon forming a MFC stack (Fig. 1). Finally, the fabrication of the individual MFC modules were completed by folding the left half to the right half along the predefined crease \#3 and applying the spray adhesive onto only the peripherals of each layer through a patterned paper stencil (Fig. 2a (viii) \& 2a (ix)). The rhombic MFC module had the area of $8 \mathrm{~cm}^{2}$. An inlet appeared on the side of the cathodic layer, directly reaching the paper reservoir layer through the 
holes in the cathodic and PEM layers (Fig. 2a (x)). This configuration covered the anodic compartment minimizing evaporation of the organic liquid to be applied. When the bacterial cells were introduced through the inlet, they were transported horizontally and vertically, filling the reservoir and anodic layers. The rapid adsorption of the hydrophilic layers (paper reservoir, carbon cloth, and anodic paper layer) promoted the cell attachment to the anode, where bacterial respiration could then transfer electrons from the organic fuels to the anode. Then, the electrons flowed through an external wire under a load to the cathode. The protons were transferred through the PEM and reduced with the oxygen and electrons travelled from the anode. During the operation, the paper reservoir, PEM, and anodic paper layer held organic fuels for an extended time during the MFC operation.

\subsection{MFC stack assembly}

We fabricated seven more of these MFC modules for a total of eight. Each MFC module has one blade on one side and two flaps on the other side (Fig. 2b (1)). First, one blade side of the MFC module \#1 was put between the double flaps of another MFC module \#2 (Fig. 2b (2)). Then, the electrical contact parts of the flaps were folded to lock the module \#2 into place (Fig. 2b (3)). In this way, the other MFC modules were added and assembled until all eight modules were locked into place to form a round frisbee (Fig. $2 b$ (4) (9). With this frisbee shape, all eight MFC modules were connected in series. For the actual application, one of the connections needed to be disconnected and to be used as the anode and cathode of the whole stack. Also, the frisbee was retractable into an eight point ninja star by holding opposite sides of the modules and pushing in towards the center (Fig. 2b (10)). Then, the series connection of the stack was disconnected while the inlets of the individual MFCs were placed into the center. The total cost of the fabricated device was about $\$ 0.7$ which can be reduced further if the expensive Nafion solution is replaced with low-cost polytetrafluoroethylene (PTFE). The size of a completely assembled device was $7.5 \mathrm{~cm}$ x $7.5 \mathrm{~cm}$ when opened, and $9 \mathrm{~cm} \times 9 \mathrm{~cm}$ when closed. 


\subsection{Inoculum}

In order to verify the feasibility of the fabricated MFC modules, a pure culture of Shewanella oneidensis MR-1 in standard L-broth medium was first used. The L-broth media consisted of 10.0 g triptone, $5.0 \mathrm{~g}$ yeast extract and $5.0 \mathrm{~g} \mathrm{NaCl}$ per litre. The cells were harvested by centrifugation to remove biomass and re-suspended in a fresh medium. Growth was monitored by measurement of the optical density at $600 \mathrm{~nm}\left(\mathrm{OD}_{600}\right)$ and the culture we used reached an $\mathrm{OD}_{600}$ of 2.0.

Ideally, our paper-based MFC stack was to be made operable by any liquid readily accessible in the local environment. Normally, the MFCs operated with wastewater or sewage sludge require a significant amount of time until they yield actual electricity. ${ }^{30-32}$ The liquid itself cannot be an energy source for the short-term applications such as disposable, paper-MFC batteries because the bacterial cells in the liquid are not fully cultured to the number enough for their power generation. That is why our previous paper-based MFC work was limited to a high concentration of the well

cultured lab inoculum. ${ }^{21-27}$ In this work, we used a wastewater sample cultured in a LB media for 24 hours at room temperature. Only $1 \mathrm{ml}$ of the wastewater was introduced in a $20 \mathrm{~mL} \mathrm{LB}$ media for the fast bacterial growth/reproduction and left in the lab. The wastewater was obtained from the Binghamton-Johnson City Joint Sewage Treatment Plant. The optical density of the culture reached an $\mathrm{OD}_{600}$ of 2.6, which was made into 2 for direct comparison with S. oneidensis cultured sample. This simple culture method can be easily applicable even in resource-limited regions. Library preparation and quality assurance/control for microbial 16s rDNA (V4 hypervariable region) amplicon sequencing of the wastewater was performed at the Microbiome Analysis Lab. at Arizona State University.

\subsection{Measurement setup}

The potential difference between electrodes was measured by a data acquisition system (NI, USB 6212). The voltages were measured every 30 seconds and recorded via a customized LabVIEW 
interface. An external resistor was connected between the anode and cathode to induce the flow of current, which was calculated via Ohm's law.

\subsection{Bacterial fixation and SEM imaging}

The paper-based MFC stack was disassembled, rinsed, and adherent bacteria on each layer were immediately fixed in $2 \%$ glutaraldehyde solution overnight at $4{ }^{\circ} \mathrm{C}$. Samples were then dehydrated by serial 5 min transfers through $50,70,80,90,95$, and $100 \%$ ethanol. Fixed samples were examined using a FESEM (Field Emission SEM) (Supra 55 VP, Zeiss).

\section{Results and discussion}

\subsection{Optimization of sample volume}

Compared with the conventional LOC devices, capillary pumping is the method of liquid transport in the paper device, and our MFC module fabricated from paper provides 3D fluidic pathways to each functional layer without the need for pumps or other equipment. Basically, the sample solution is transported to four layers of the MFC module; starting from paper reservoir to paper PEM, and carbon cloth. It is critical to maximize the uniformity of the bacterial cells' distribution over the carbon cloth layer through the reservoir layer (Whatman \#1, pore size: $10 \mu \mathrm{m}$ ) for high power output of the MFC. Moreover, the sufficiency of organic fuels in the other layers needs to be ensured for relatively long operation time. Based on our previous research, the bacterial cells were successfully transported even in much smaller pore size paper (Whatman \#5,

pore size: $2.5 \mu \mathrm{m}) .{ }^{29}$ However, too small volume of the sample will not generate enough capillary force to move the liquid and bacterial cells to the end of each layer while too large volume may cause leaking of the solution making it malfunctional such as creating a short circuit between the anode and cathode. Therefore, there is a need to optimize pipetting volume of solutions 
introduced onto the MFC module. For this study, we prepared the device samples consisting of four layers (paper PEM, paper reservoir, carbon cloth, and paper cover layer for the anode). Since the top paper PEM (Whatman \#410) among all four layers has the lowest flow rate due to their smallest pore size $(1 \mu \mathrm{m})$, the flow of the colored ink over this PEM layer can be a good indication to understand the sample transportation through all four layers during wet-out. The spray adhesive covered all over the anodic paper blocked the solution penetration from the carbon cloth. As shown in Fig. 3, 30 $\mathrm{L}, 50 \mu \mathrm{L}, 80 \mu \mathrm{L}, 100 \mu \mathrm{L}$ and $150 \mu \mathrm{L}$ of the solution volume were not enough to wick the entire PEM along with all other two layers (paper reservoir and carbon cloth) even after $5 \mathrm{~min}$. The sufficiency of the sample was obtained with the pipetting volume of $200 \mu \mathrm{L}$ within 2 min. Although the bacterial distribution in the anode layer still needs to be investigated, these experimental results at least show the sufficiency of the organic fuels for single MFC module. SEM images to be discussed in the section 3.4 partly supported that the bacterial cells reached the middle of the layers.

\subsection{Bioelectricity generation from individual MFC modules}

As shown in Fig. 4a, we first tested individual MFC modules with two inoculum samples; $S$. oneidensis and wastewater. We dropped $200 \mu \mathrm{L}$ of the samples on the inlet and the output voltage between the anode and the cathode was recorded with or without an external resistor, $2 \mathrm{k} \Omega$ (Fig. 4b). All experiments were repeated eight times with different MFC modules. The open circuit voltages gradually increased over $5 \mathrm{~min}$ and reached saturation. Given that the time required for wicking all the MFC layers with $200 \mu \mathrm{L}$ solution takes only $2 \mathrm{~min}$, it must be noted that additional 3 min for the full performance of the MFC on paper is relatively short for bacterial acclimation and accumulation compared to the conventional MFCs. This is attributed to the ability of all four layers to rapidly wick the bacterial cells through capillary action and start their metabolic/extracellular electron transfer processes. Then, the external resistor was connected 
between the anode and cathode to monitor current generation. The output voltage and current from pure-cultured $S$. oneidensis was higher than those of wastewater with mixed bacterial community. Also, Fig 4c and 4d shows polarization curves and power outputs of the MFC module with S. oneidensis (Fig. 4c) and wastewater (Fig. 4d). The data were drawn based on the values recorded at a given external resistance $(500 \mathrm{k}, 300 \mathrm{k}, 100 \mathrm{k}, 50 \mathrm{k}, 15 \mathrm{k}, 5 \mathrm{k}, 2 \mathrm{k}$, and $670 \Omega)$. The maximum power densities were $13.6 \mu \mathrm{W}$ for $S$. oneidensis and $8.19 \mu \mathrm{W}$ for wastewater, respectively, at $5 \mathrm{k} \Omega$. The overall performance of the MFC with $S$. oneidensis was better than that with wastewater. This is probably because the wastewater includes many other non-electrogenic bacterial species (Fig. 1S). In order to provide insight into the microbial diversity of the sample, bacterial 16s rDNA gene libraries were examined. The qualified sequences were assigned down to the phylum, class, family and genus levels. Only three phyla dominated in the wastewater sample (Proteobacteria, Bacterioidetes, and Firmicutes). The most dominant phyla was the Proteobacteria (81\%), including 67\% Alphaproteobacteria, 8\% Betaproteobacteria, $2 \%$ Deltaproteobacteria, 2\% Epsilonproteobacteria, and 1\% Gammaproteobacteria. It was reported that Alphaproteobacteria dominated a community with a river sediment inoculum enriched with a low concentration of glucose and glutamate while Gammaproteobacteria and Deltaproteobacteria might be involved in current generation of the MFCs. ${ }^{33,34}$ The second most dominant phylum in all microcosms was the Bacterioidetes (13\%), within which the Bacterioidia (12\%) was the most abundant class. The dominant taxa included the genus of Bosea, Unclassified Porphyromonadaceae, Unclassified Bacteroidales, and Dechloromonas. In our wastewater sample, there were not that many electrogenic bacterial species. For further studies, we need to find out what type of electrogenic bacteria is contained in various types of liquid in nature or in our environment, and how much power can be extracted from each. This research will reveal electrogenic bacterial communities in our environment and enable us to develop various strategies to use the bacteria-powered battery in resource-limited settings. 


\subsection{Bioelectricity generation from the MFC stack}

The performance of the MFC modules even with the simply prepared wastewater was highly comparable to those with usual lab grown inoculum, which confirms that our paper-based MFC battery can be potentially used even in resource limited regions. In order to improve the power output for actual applications, eight MFC modules were assembled to form a ninja star based battery stack. For the sample introduction, we transformed the device into the sharp shuriken. After $1.6 \mathrm{~mL}$ of the solution in total $(200 \mu \mathrm{L}$ per module) was dropped on inlets of the MFC stack, we waited $5 \mathrm{~min}$ for the bacterial transportation/attachment/acclimation on each device layer and then transformed the device back to the round frisbee in order to (i) connect modules in series and (ii) expose the cathode to the air. If the MFC modules are pre-connected in series before the bacterial cells are settled down on the anode, the power output cannot be maximized due to the voltage reversal issue in some of the MFC modules. The voltage reversal has been one of the main challenges of the MFC arrays during the high current production. ${ }^{35-37}$ Our device configuration can be an efficient solution to this issue because the battery stack is completely disconnected during the bacterial introduction and can take some time while the cells are fully accumulated on the anode. It will not be until the stack is retracted into the round frisbee that individual MFC modules are connected in series. The open circuit voltage of the stack under the frisbee shape was $2.76 \mathrm{~V}$, which was equal to the sum of the individual MFC modules' voltages on averages, $0.345 \mathrm{~V}$ (Fig. 5a). When the frisbee was transformed into the shuriken, the voltage dropped to the zero. Repeated transforming cycles of the stack continued to show good reproducibility of voltage generation, which also suggested that the device was robust and reliable. With more cycles, the voltage generation was slightly reduced with shorter rising time.

In addition, the battery stack provided the desired values of current and voltage for powering a red LED (HLMPP156, Digikey) for over 20 min without power management interface circuits (Fig. 5b). While the LED was being illuminated by the frisbee stack, the voltage was also 
recorded for the duration of the operation. Upon the LED connection, the voltage instantly dropped to $1.7 \mathrm{~V}$ and gradually decreased. When the voltage output was less than $1.5 \mathrm{~V}$, the LED was turned off. The LED never flashed with LB media only without the bacterial cells, suggested that the power output was generated from the bacterial metabolism.

\subsection{SEM images}

The bacterial cell's movement in the battery stack can be further explained by analysing electron microscope examinations of the different layers (Fig. 6). After the MFC stack powered the LED, it was disassembled and three layers (paper PEM, paper reservoir, and carbon cloth) were prepared for the SEM images. On top of the paper PEM which physically contacted with the aircathode, no bacterial cells were found, indicating that the cells could not pass through the small pore size paper and this layer might be performed as a good PEM. On the other hand, very densely packed bacterial cells were observed on the paper reservoir, while a less number of cells was found on the carbon anode. The cells shown on both layers suggest that the sample introduced from the inlet was well transported into the bottom layer of the anodic carbon layer. Further studies need to be done to investigate the uniformity of the bacterial cells throughout the carbon cloth.

\section{Conclusion}

There are various types of paper-based batteries including Li-ion batteries, superconductors, mechanical energy harvesters, electrochemical batteries, and biofuel cells. The choice of the type depends on the application. For high-power portable electronic applications, Li-ion batteries or supercapacitors are good power source while mechanical energy harvesters are suitable for wearable electronics. Electrochemical batteries will be good candidates for paper-based microfluidic devices. Above all, the paper-based biofuel cells are more attractive due to environmentally friendly or economically disposable features while the 
electrode or electrolyte used in other battery types is unstable, explosive, flammable, and environmentally hazardous. Moreover, their battery configurations compared to that of the biofuel cell require many functional layers to be deposited onto paper, and this increases design complexity and fabrication processes which, in turn, makes them high-cost tools. However, the previous paper-based biofuel cells showed relatively low power generation and many operational limitations. In this work, we created a simply transformable origami paper-based biofuel cell stack for their operation and performance. Microorganism-containing liquid was added into the inlets centered in the middle of the closed battery stack (sharp shuriken), though which the liquid was transported into each MFC modules. The hydrophilic layers in the device rapidly wicked the liquid through capillary action and allowed for a faster bacterial accumulation and acclimation on the anodic layer. For operation, the MFC stack was opened (round frisbee shape) to connect each MFC module in series and expose all air-cathode to the air for their cathodic reactions. The battery stack provided desired values of current and voltage to power a red LED for more than $20 \mathrm{~min}$, indicating that the device can probably power actual portable applications. For example, the stack can power the fluorescent assay for monitoring the target analyte. This novel MFC stack will be a simple, low-cost, easy-to-use, and disposable power supply for potentially one-time use POC diagnostic devices in resource-limited settings.

\section{Acknowledgments}

This work is supported by NSF (ECCS \#1503462).

\section{References}

1 C.H. Ahn, J. Choi, G. Beaucage, J.H. Nevin, J. Lee, A. Puntambekar, J.Y. Lee, Proceedings of the IEEE, 2004, 92, 154-173. 
2 B. Samel, V. Nock, A. Russom, P. Griss, G. Stemme, Biomed Microdevices, 2007, 9, 61-67.

3 S. Choi, M. Goryll, L.Y.M. Sin, P.K. Wong, J. Chae, Microfluid Nanofluid, 2011, 10, 231-247.

4 C.D. Chin, V. Linder, S.K. Sia, Lab Chip, 2012, 12, 2118-2134.

5 L.R. Volpatti, A.K. Yetisen, Trends Biotechnol, 2014, 32, 347-350.

6 S. Choi, Biotechnology Advances, 2016, 34, 321-330.

7 B.E. Rittmann, Biotechnology and Bioengineering, 2008, 100, 203-212.

8 B.E. Logan, Nat Rev Microbiol, 2009, 7, 375-381.

9 D.R. Lovley, Annu Rev Microbiol, 2012, 66, 391-409.

10 U. Schröder, F. Harnisch, L.T. Angenent, Energy \& Environmental Science, 2015, 8, 513-519.

11 C.I. Torres, A.K. Marcus, H. Lee, P. Parameswarn, R. Krajmalnik-Brown, B.E. Rittmann, FEMS Microbiol Rev, 2010, 34, 3-17.

12 A.P. Borole, G. Reguera, B. Ringeisen, Z. Wang, Y. Feng, B.H. Kim, Energy \& Environmental Science, 2011, 4, 4813-4834.

13 T. Ewing, P.T. Ha, J.T. Babauta, N.T. Tang, D. Heo, H. Beyenal, Journal of Power Sources, 2014, 272, 311-319.

14 B.E. Logan, Appl Microbiol Biotechnol, 2010, 85, 1665-1671.

15 W. Li, H. Yu, Z. He, Energy \& Environmental Science, 2014, 7, 911-924.

16 S. Choi, Biosensors and Bioelectronics, 2015, 69, 8-25.

17 H. Lee, S. Choi, Lab Chip, 2015, 15, 391-398.

18 F. Qian, D.E. Morse, Trends in Biotechnology, 2011, 29, 62-69.

19 H. Wang, A. Bernarda, C. Huang, D. Lee, J. Chang, Bioresource Technology, 2011, 102, 235243.

20 A. Han, H. Hou, L. Li, H. S. Kim, P. de Figueiredo, Trends in Biotechnology, 2013, 31, 225232.

21 H. Lee, S. Choi, Nano Energy, 2015, 15, 549-557. 
22 A. Fraiwan, S. Choi, Physical Chemistry Chemical Physics, 2014, 16, 26288-26293.

23 A. Fraiwan, H. Lee, S. Choi, IEEE Sensors Journal, 2014, 14, 3385-3390.

24 T.H. Nguyen, A. Fraiwan and S. Choi, Biosensors and Bioelectronics, 2014, 54, 640-649.

25 A. Fraiwan, S. Mukherjee, S. Sundermier, H.-S. Lee, S. Choi, Biosensors and Bioelectronic, 2013, 49, 410-414.

26 G. Choi, D. Hassett, S. Choi, Analyst, 2015, 140, 4277-4283.

27 A. Fraiwan, S. Choi, Biosensors and Bioelectronics, 2016, 83, 27-32.

28 G. Choi, S. Choi, Analyst, 2015, 140, 5901-5907.

29 G. Choi, S. Choi, Sensors \& Actuators:B. Chemical, 2016, doi:10.1016/j.snb.2015.11.127 (in print)

30 Z. Wang, J. Ma, Y. Xu, H. Yu, Z. Wu, Journal of Power Sources, 2013, 235, 280-288.

31 G. Zhang, Q. Zhao, Y. Jiao, K. Wang, D. Lee, N. Ren, Water Research, 2012, 46, 43-52.

32 C. Abourached, K.L. Lesnik, H. Liu, Bioresource Technology, 2014, 166, 229-234.

33 Y. Zhang, B. Min, L. Huang, I. Angelidaki, Applied and Environmental Microbiology, 2009, 75, 3389-3395.

34 Y. Sang, L. Xiao, I. Jayamani, Z. He, A.M. Cupples, Journal of Microbiological Methods, $2015, \mathbf{1 0 8}, 4-11$.

35 S. Choi, J. Chae, Sensors and Actuators: A. Physical, 2012, 177, 10-15.

36 J. An, J. Sim, H.S. Lee, Journal of Power Sources, 2015, 283, 19-23.

37 Y. Kim, M.C. Hatzell, A.J. Hutchinson, B.E. Logan, Energy \& Environmental Science, 2011, 4, $4662-4667$ 


\section{Figure and Table captions}

Figure 1: Conceptual device photo-images of the origami paper-MFC stack. Each MFC module is prepared as a blade for the eight point ninja star. The sharp shuriken (a) can be transformed into the round frisbee (b) for operation as a power source.

Figure 2a: Fabrication processes of each MFC module (i ix) and its operating principle (x). Each layer was carefully assembled and sandwiched by using adhesive spray. The hydrophobic wax boundaries were patterned all over the peripherals of the device to hold the liquid without leakage. (i) the patterned paper substrate with the wax boundaries and predefined cut/creases was prepared, (ii) the bottom left of the paper was folded back to the right and folded up along the predefined creases, (iii) the carbon cloth anode was placed and pasted on the right of the paper, (iv) the paper reservoir layer was attached on top of the anode, (v) Nickel was sprayed on the left side of the paper substrate and then air-dried thoroughly at room temperature for 2 hours, (vi) an activated carbon-based catalytic solution was applied on the Nickel electrode to construct an air-cathode, (vii) the paper with very small pores was attached over the reservoir layer as a PEM, (viii) \& (ix) the cathode part was folded to the right to form a completed MFC module. The copper tape was used for all electrical contacts. (x) the schematic diagram of the cross section of the MFC module (a-a' shown in (h)).

Figure 2b: Assembly processes of the MFC stack. (1) individual MFC modules were prepared with electrical contacts, (2) one blade of the MFC module \#1was placed between the double flaps of another MFC module \#2, (3) the parts of two flaps of the module \#2 were folded and pulled down so the module \#2 locked at the end, (4 9) in this way, the module \#3 \#8 were assembled and locked into place to form a round frisbee. We ensured that all the electrical connections were made. (10) the frisbee can be transformed into the eight point ninja star.

Figure 3: Images on the optimum pipetting volume of solutions on the MFC module. The MFC modules without the cathodic parts were prepared to determine the optimal sample volume. (a) $30 \mu \mathrm{L}$, (b) $50 \mu \mathrm{L}$, (c) 
$80 \mu \mathrm{L}$, (d) $100 \mu \mathrm{L}$, (e) $150 \mu \mathrm{L}$, and (f) $200 \mu \mathrm{L}$ of the blue ink were introduced on the inlets of difference devices. (g) the schematic diagram of the cross section of the device (a-a' shown in (f)).

Figure 4: Bioelectricity generation from individual MFC modules. (a) photograph of the test setup, (b) output voltage profiles with or without an external resistor $(2 \mathrm{k} \Omega)$, and polarization curves/power outputs of the devices with (c) S. oneidensis and (d) wastewater.

Figure 5a: Battery stack operation. The stack was transformed (a) into the shuriken for the sample introduction, or (b) into the frisbee for the operation. (c) The device transformation was repeated. (d) Open circuit voltage profiles from the stack during the repeated transformation cycles.

Figure 5b: Output voltage of the battery stack when an LED was connected across the stack in series. The LED was lightened for more than 20 min.

Figure 6: SEM images of the paper PEM, paper reservoir, and carbon cloth anode used in one of the MFC modules after the LED lighting. Scale bar is $5 \mu \mathrm{m}$. 
Closed battery stack for

inoculum addition

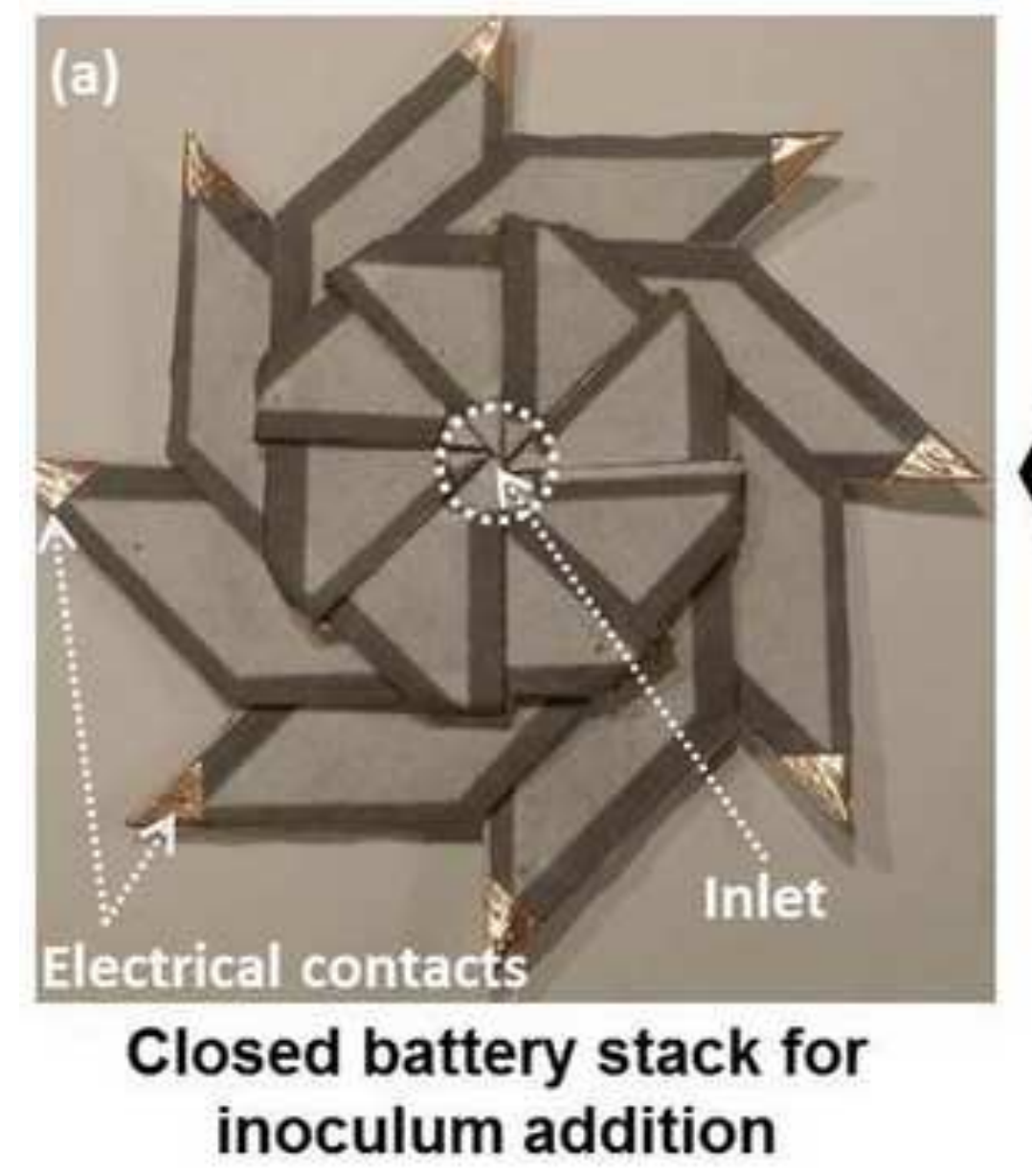

Opened battery stack

for operation

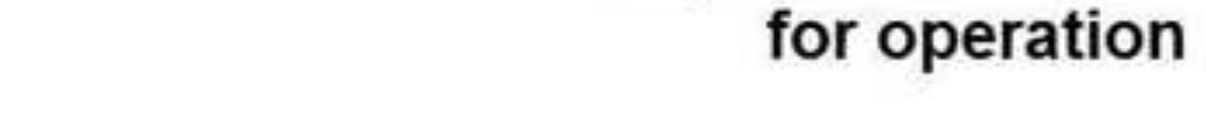

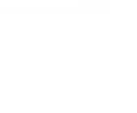

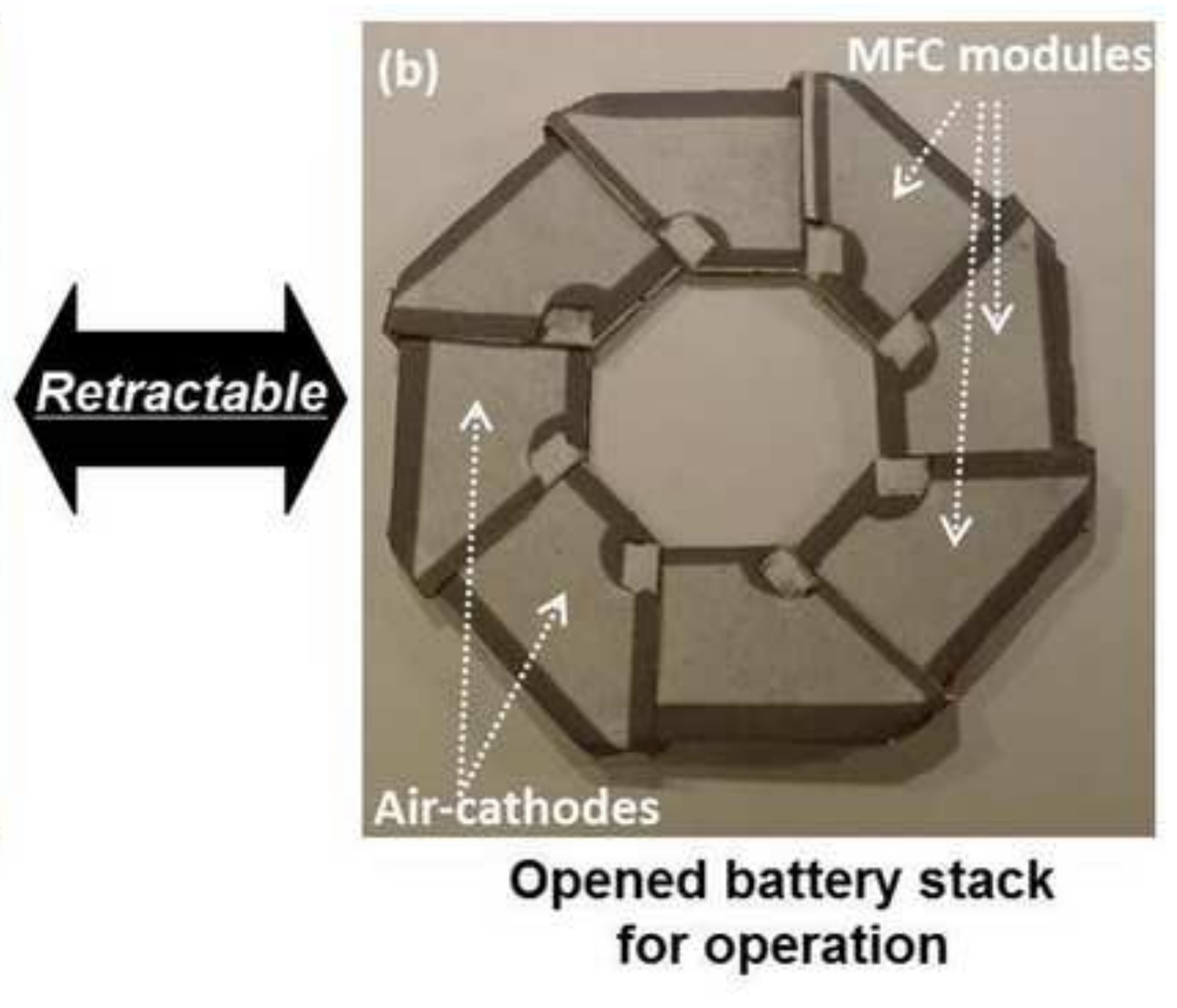
(n) mannam

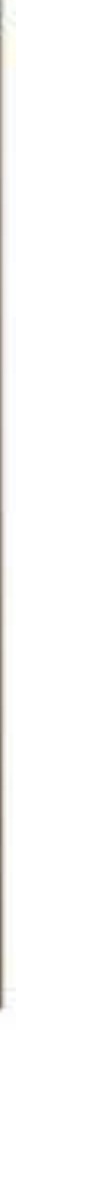

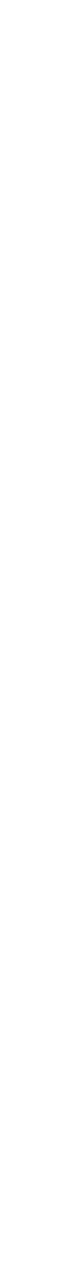


(a) (i)

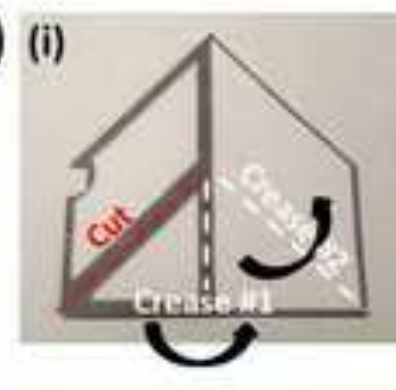
boundaries
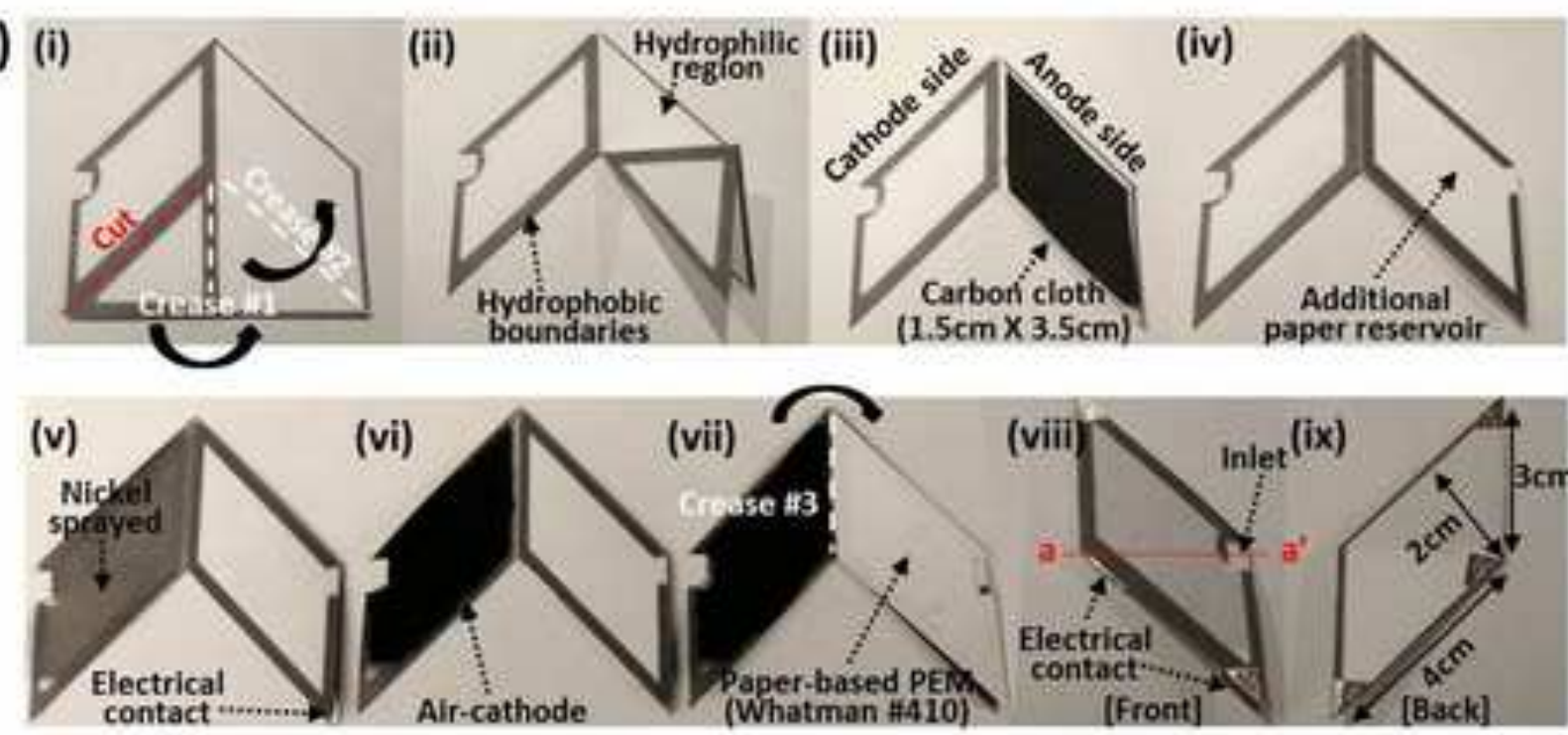

(vi)

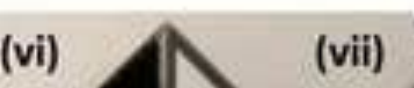
paper reservoir

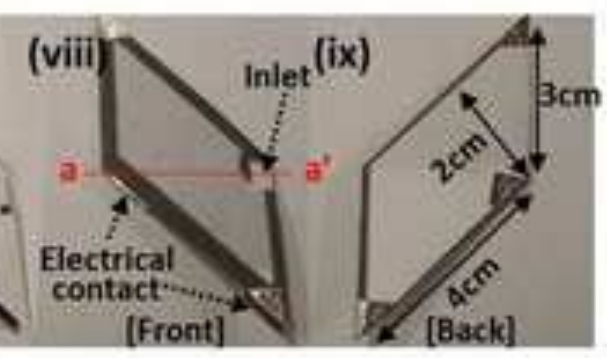

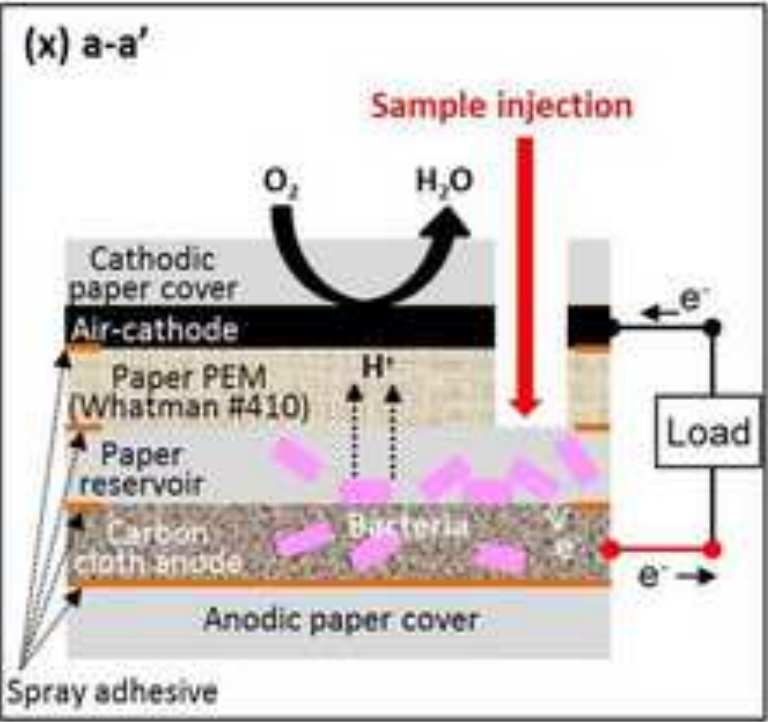

(b) (1)
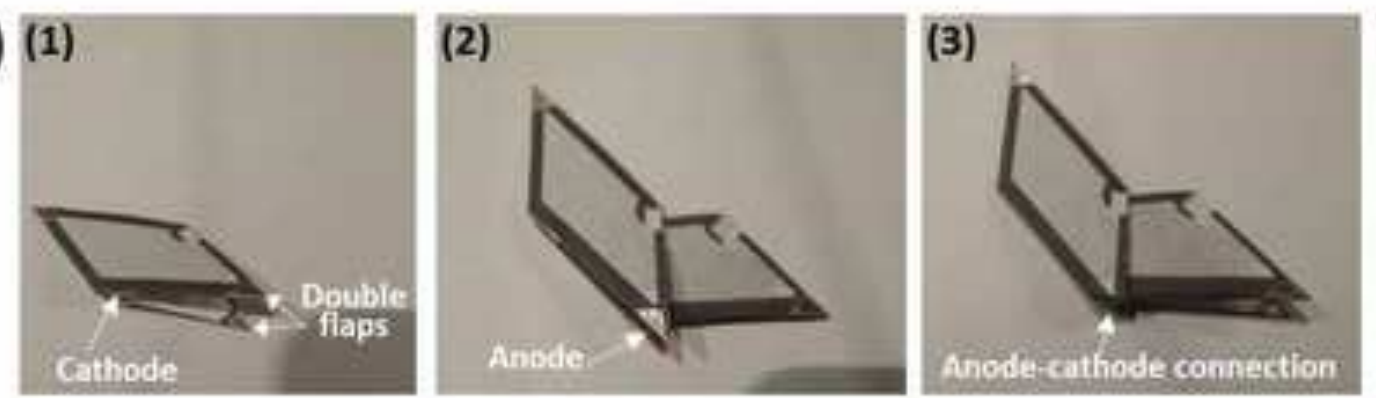

(6)

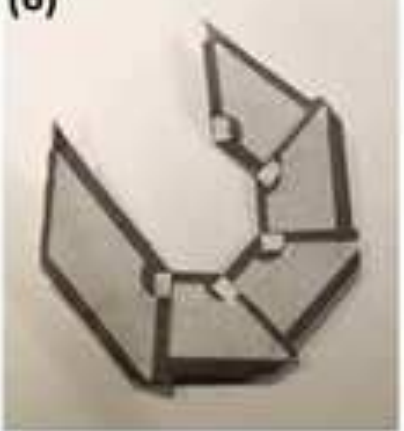

(7)

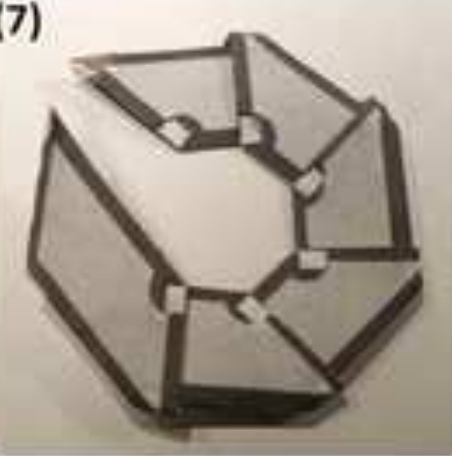

(8)

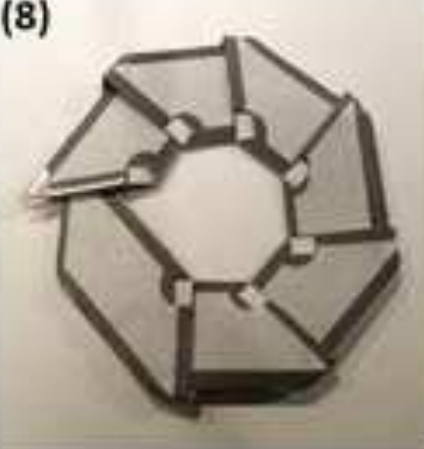

(4)

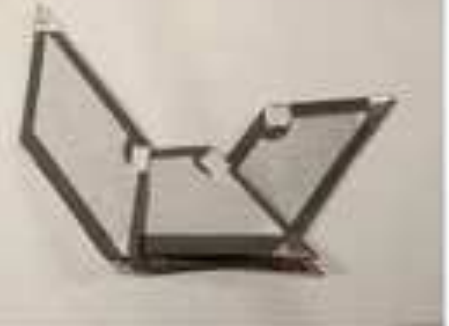

(9)

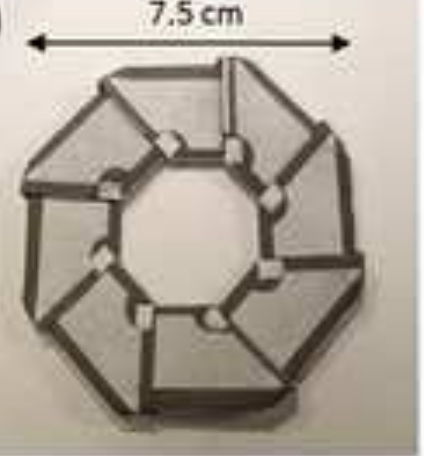

(5)

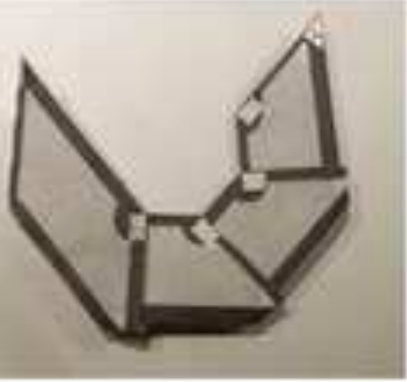

(10)

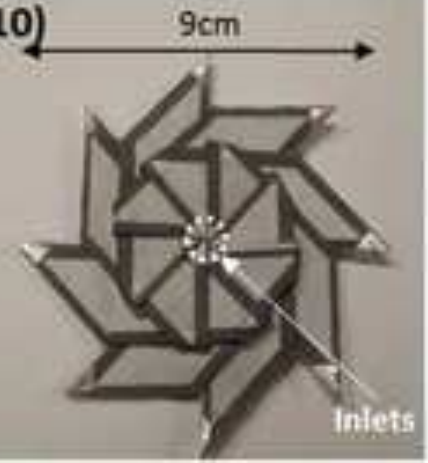




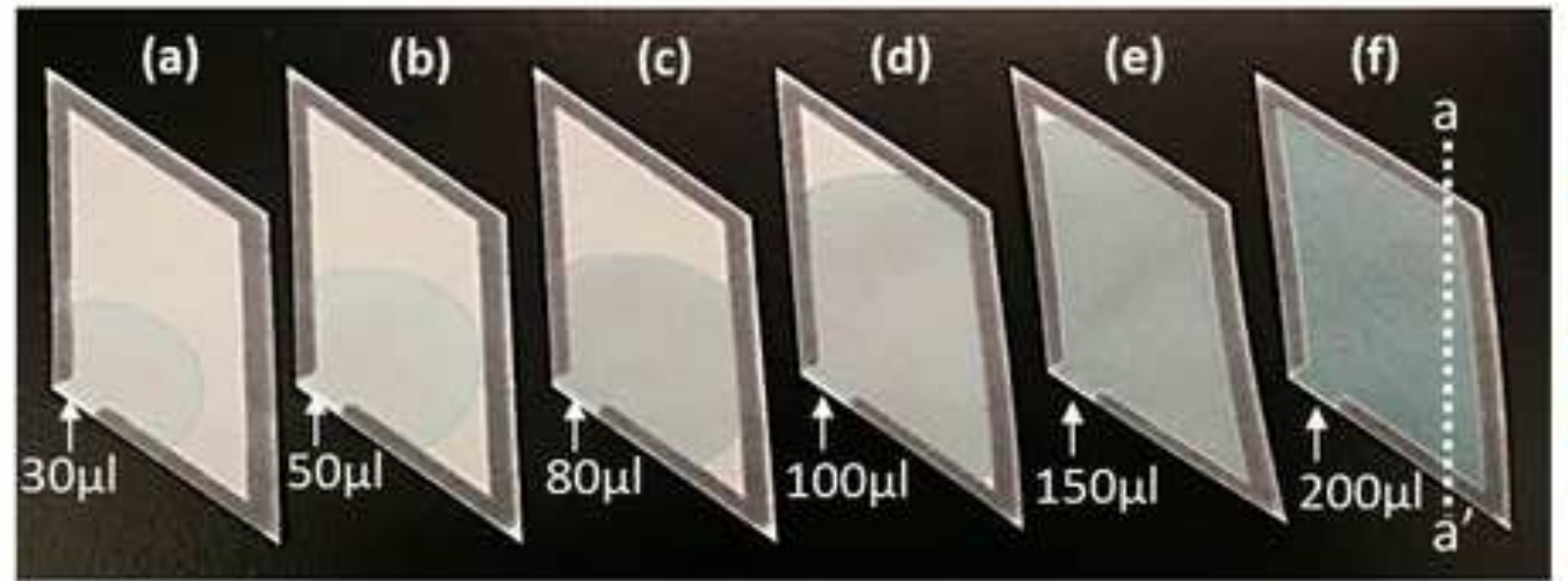

(g) a-a

Blue Ink injection

Paper PEM (Whatman \#410)

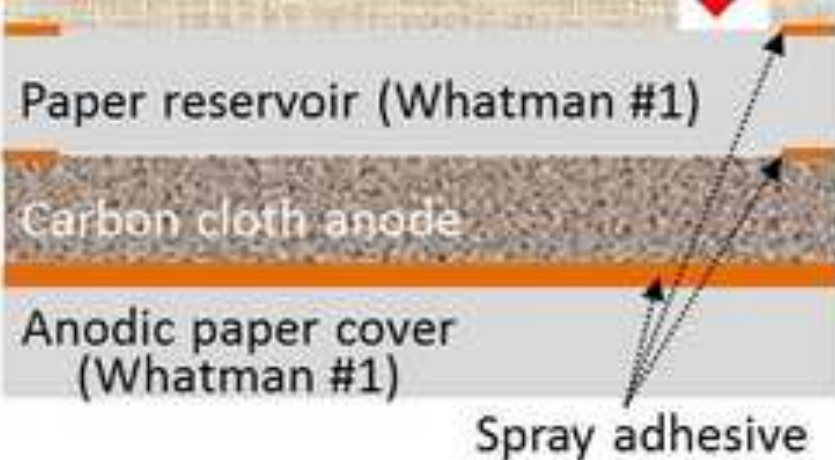


(a)
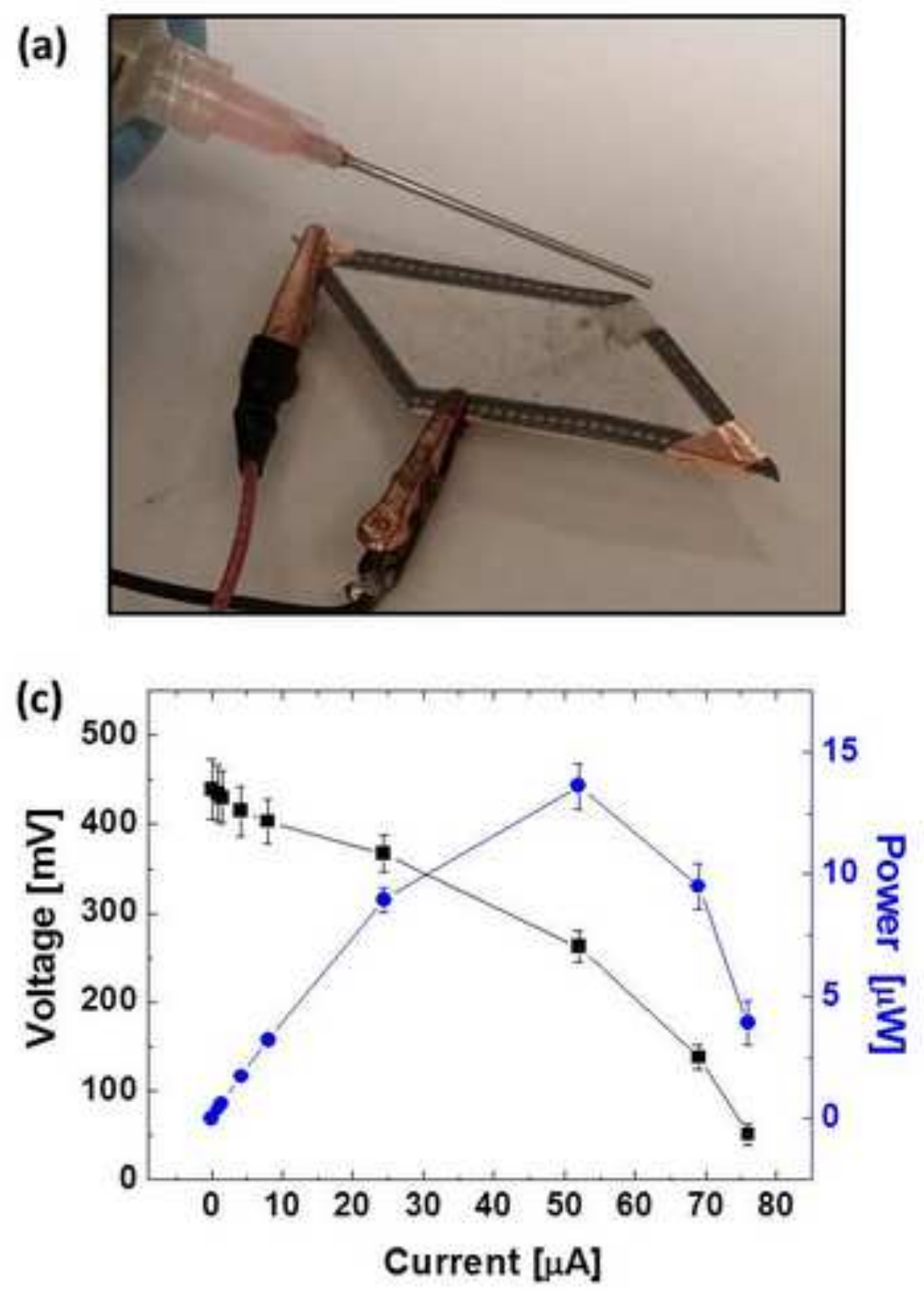

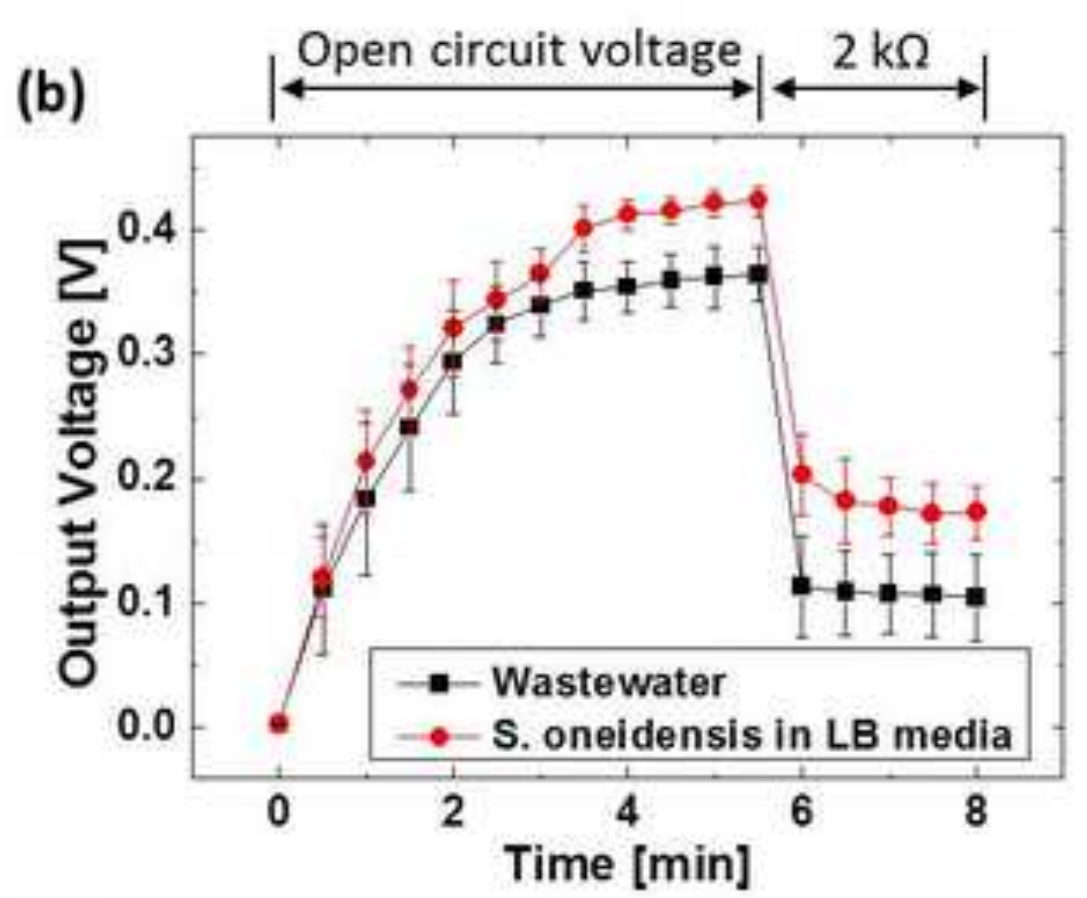

(d)

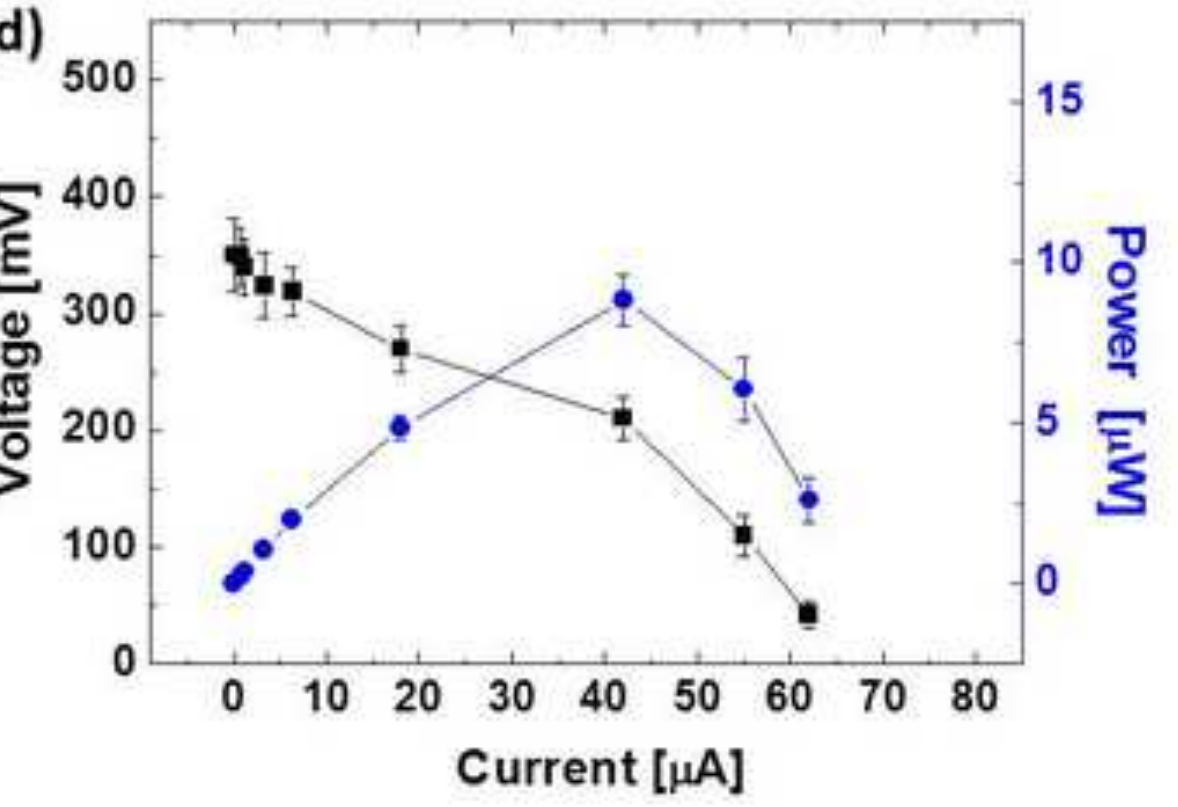



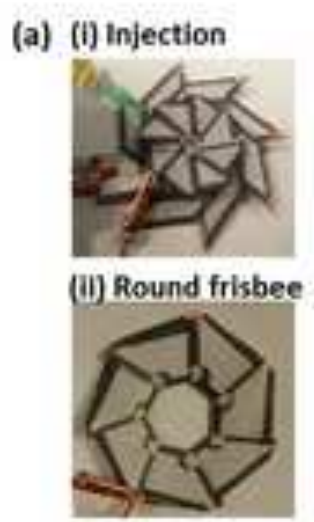

(iii) Sharp shuriken

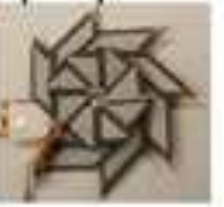

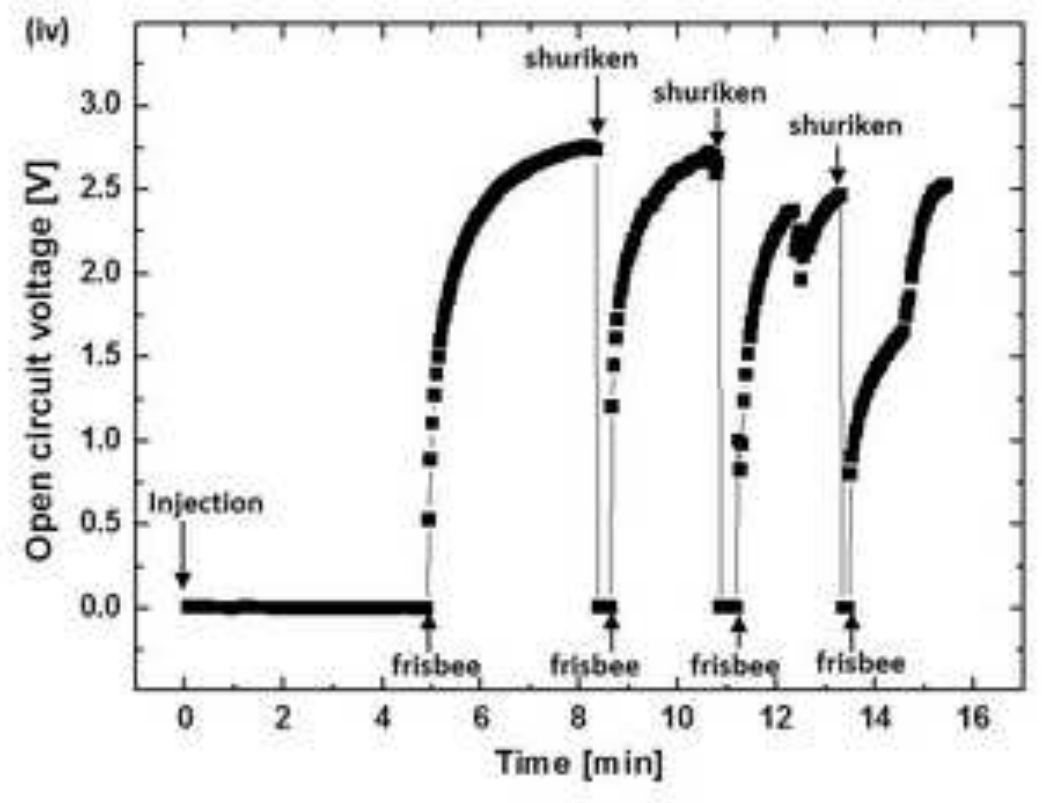

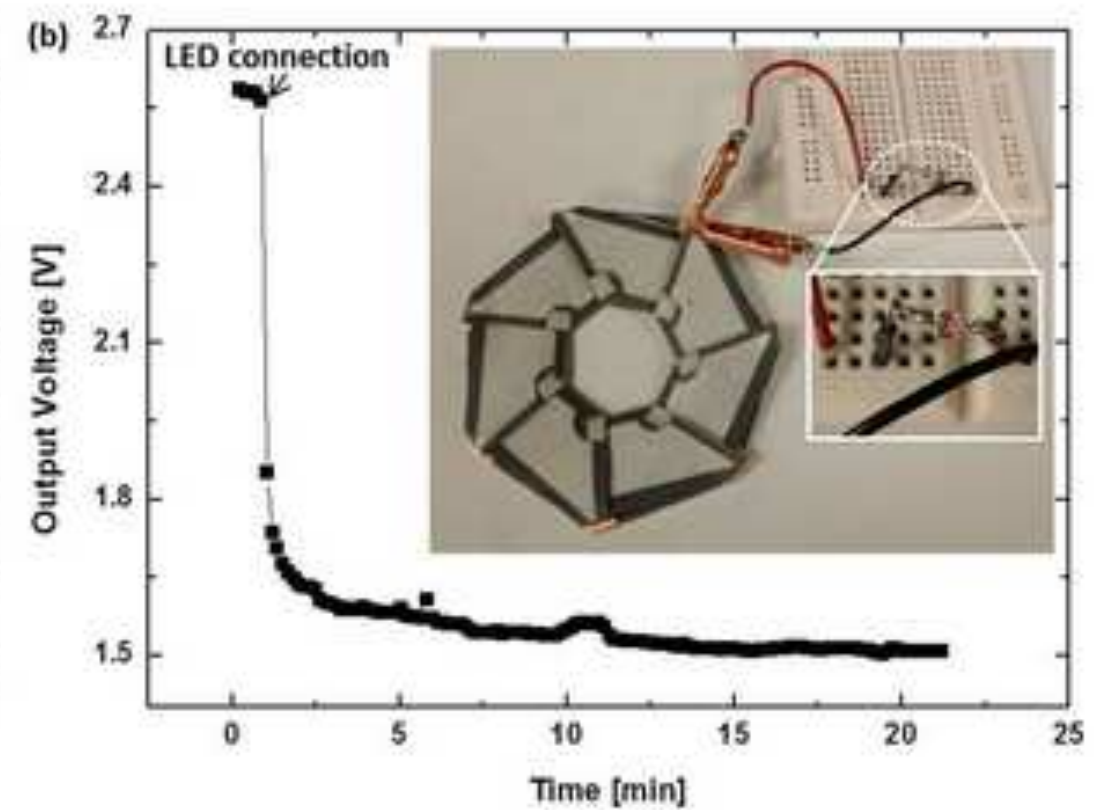


Sample injection
Scanning electron microscope

(1) (2)

(2) (3)

Cathodic paper cover (Whatman \#1) Air-cathode

Paper PEM (Whatman \#410)

Paper reservoir (Whatman \#1) cearboholothanodes Anodic paper cover (Whatman \#1)
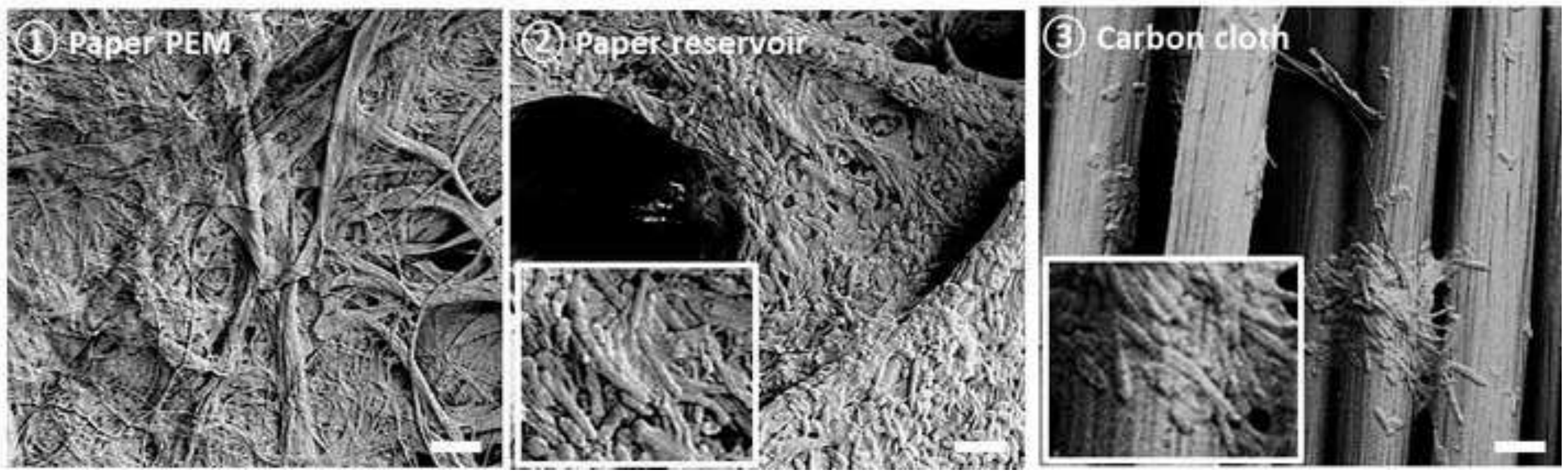